\title{
Wavefront-sensor-induced beam size error: physical mechanism, sensitivity-analysis and correction method
}

\author{
Wouter D. Koek*, Erwin J. van Zwet \\ TNO, Stieltjesweg 1, 2628CK, Delft, The Netherlands
}

\begin{abstract}
When using a commonly-used quadri-wave lateral shearing interferometer wavefront sensor (QWLSI WFS) for beam size measurements on a high power $\mathrm{CO}_{2}$ laser, artefacts have been observed in the measured irradiance distribution. The grating in the QWLSI WFS not only generates the diffracted first orders that are required for introducing the shear, but also diffracts significantly into higher orders. Consequently, in the few millimeters of free space propagation between the QWLSI WFS grating and its imaging device, the beam size may increase significantly (particularly for infrared wavelengths). This error is typically not accounted for in the subsequent processing of measurement data.
\end{abstract}

To gain insight in this undesirable behavior, physical models of the QWLSI WFS using both complex wave propagation and analytic propagation of the D4sigma beam diameter (and its associated $\mathrm{M}^{2}$ ) throughout the system have been developed. These models show excellent agreement to experimental data, and indicate that in typical situations the sensor-induced beam size error can easily be $40 \%$ or more.

Although the QWLSI WFS may not originally be intended for beam size measurements, in most industrial applications cost- and volume limitations will often lead to multiple use of sensor data. To aid in the adequate implementation of a QWLSI WFS for determining beam size, the dependence of the sensor-induced beam size error on various system parameters has been determined (e.g. incoming beam size, grating-to-imager distance, grating geometry, wavelength). Using the presented models and guidelines, the sensor-induced beam size error may be minimized and corrected for.

Keywords: beam size error, quadri-wave lateral shearing interferometer, wavefront sensor, QWLSI WFS, wavefront propagation, D4sigma, beam diameter, sensor-induced beam size error

\section{INTRODUCTION}

Over the past years we have encountered multiple organizations, which for a variety of applications, use a quadri-wave lateral shearing interferometer wavefront sensor (QWLSI WFS ${ }^{1}$ to perform measurements on a high-power $\mathrm{CO}_{2}$-laser beam. These lasers, and thus also the associated metrology systems, are used in industrial settings. In industrial applications it is frequently observed that cost- and volume limitations will lead to multiple use of sensor data. Although the QWLSI WFS may not originally be intended for beam size measurements, it is our experience that it is commonly used for this purpose. Furthermore, also for cost reasons, it may be decided that multiple beams are measured onto a single sensor, thereby reducing the spatial extent of a single beam.

A commercially available QWLSI WFS is the SID4 LWIR ${ }^{2}$. The analysis and results in this study relate to this specific instrument.

When analyzing 1D-binned raw QWLSI sensor data a sloped foot of the irradiance distribution was observed which, given the prior knowledge about the system, was not present in the input beam. As shown in Figure 1, when looking at the original raw image we observed 'bleeding' along two orthogonal axes which were not aligned with the pixel grid of the image array. It was suspected that these artefacts are introduced by the QWLSI WFS itself. Even though these artefacts contain only a small amount of energy, they may still cause a significant error in parameters (such as beam diameter) that are determined using these images.

In this study we investigate the magnitude of this sensor-induced beam size error, and its dependence on various system parameters (e.g. incoming beam size, grating-to-imager distance, grating geometry, wavelength).

*wouter.koek@tno.nl

High-Power Laser Materials Processing: Lasers, Beam Delivery, Diagnostics, and Applications IV, edited by Friedhelm Dorsch, Proc. of SPIE Vol. 9356, 935604 · C) 2015 SPIE

CCC code: $0277-786 X / 15 / \$ 18 \cdot$ doi: $10.1117 / 12.2077087$ 


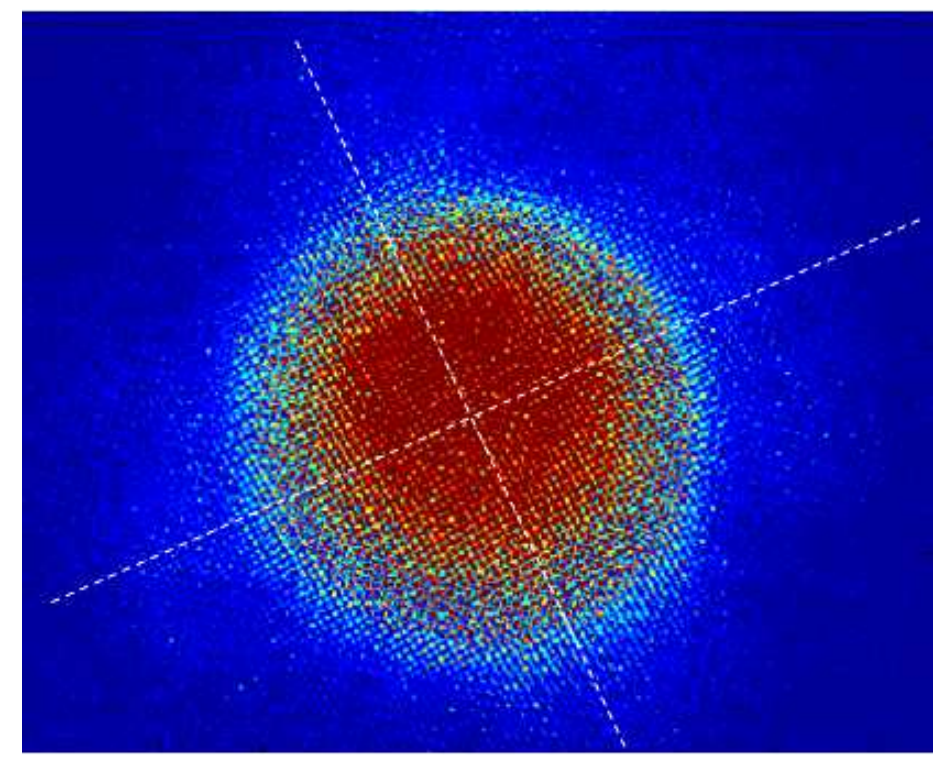

Figure 1. Selected region of raw QWLSI WFS data (i.e. the interferogram). The dynamic range of the image has been adjusted such that the lower irradiance levels are observed. The data shows a central beam that has been smeared out along the indicated axes.

\section{THEORY AND MODELING}

\subsection{QWLSI WFS}

The QWLSI WFS contains a 2D periodic phase amplitude grating which has been specifically designed such that the energy is mainly concentrated in the central orders ${ }^{3}$. As is shown in Figure 2, the ratio of clear aperture $a$ to the grating period $d$ fulfils $a=2 / 3 * d$, and $\pi$ phase steps occur between subsequent apertures (effectively imposing a second grating with pitch $d^{\prime}=2 \cdot d$ ).

Typically the grating period $d$ is chosen such that it corresponds to $4 \mathrm{x}$ the pixel size of the imager that is used to capture the resulting interferogram ${ }^{1}$. For the commercially available QWLSI WFS that can be used to measure radiation from a $\mathrm{CO}_{2}$-laser $(\lambda \sim 10.6 \mu \mathrm{m})$ a microbolometer-array with a pixel pitch of $25 \mu \mathrm{m}$ is used, and the grating period $d$ is thus 100 $\mu \mathrm{m}$. In this case the longitudinal spacing between the grating and the imager array is chosen to be $\sim 4 \mathrm{~mm}$.

When determining beam parameters (such as the $\mathrm{D} 4 \sigma$ or $2^{\text {nd }}$ order moment beam diameter) ${ }^{4}$ either raw or processed QWLSI WFS data can be used. The processing of the raw data (i.e. interferogram) is performed by dedicated software from the WFS manufacturer, and yields two-dimensional intensity and phase data at a resolution that corresponds to $d$.

Due to the seemingly higher spatial resolution of the raw intensity data, users of a QWLSI WFS frequently seem to have a preference to use this raw data for determining beam parameters where the phase data is not of interest. This raw data is however affected by the sensor induced artefacts that have been shown in Figure 1.

Alternatively one may use the processed data for determining the beam parameters. When processing the data, the software imposes a mask onto the raw data. The size of this mask can be selected by the user. Figure 3 shows the intensity images that were retrieved using user-selected mask radii of 100 pixels and 150 pixels. Figure 3 shows that in the reconstruction process a background intensity is introduced, where actually no energy is present. As a result the beam diameter becomes dependent on an arbitrary choice of mask size, which is obviously undesirable. As shown in Figure 3, when increasing the mask radius by $50 \%$, the $\mathrm{D} 4 \sigma$ beam diameter increases by $35 \%$ (D4 $\sigma_{\mathrm{avg}} 42.8 \mathrm{px} \rightarrow 57.7 \mathrm{px}$ ).

Throughout the remainder of this paper we will assume that beam diameter is determined using the raw interferogram. 


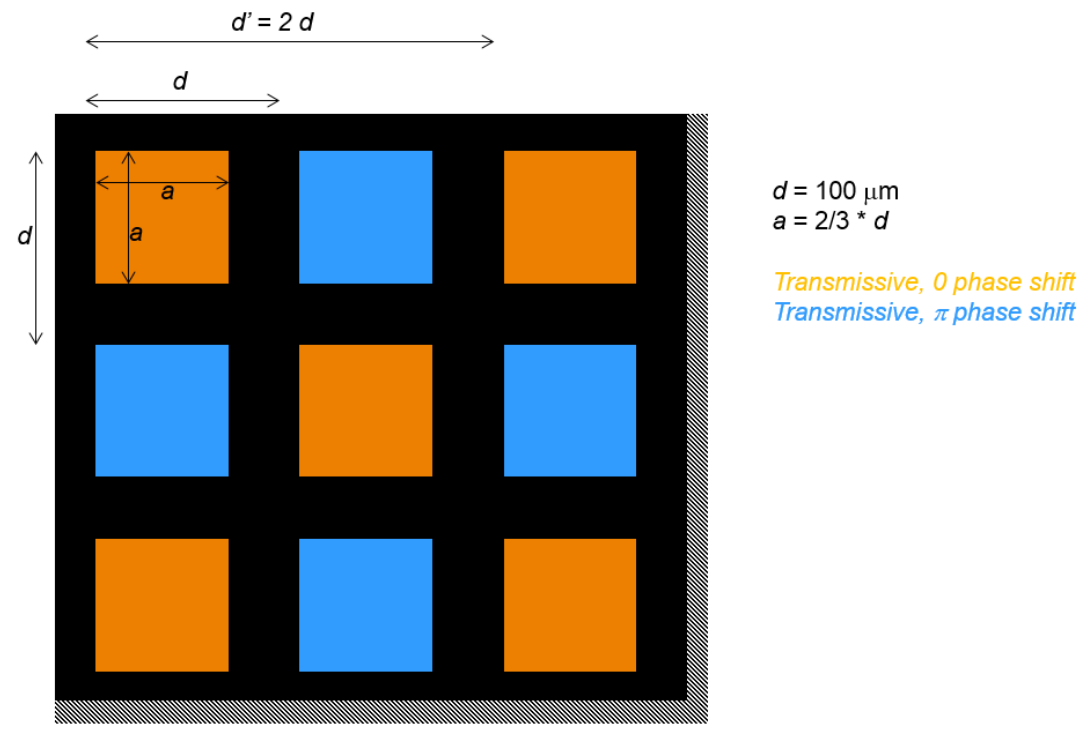

Figure 2. Schematic representation of the phase amplitude grating employed in the QWLSI WFS. The ratio of clear aperture $a$ to the grating period $d$ fulfils $a=2 / 3 * d$, and $\pi$ phase steps occur between subsequent apertures (effectively imposing a second grating with pitch $d^{\prime}=2 \cdot d$ ).
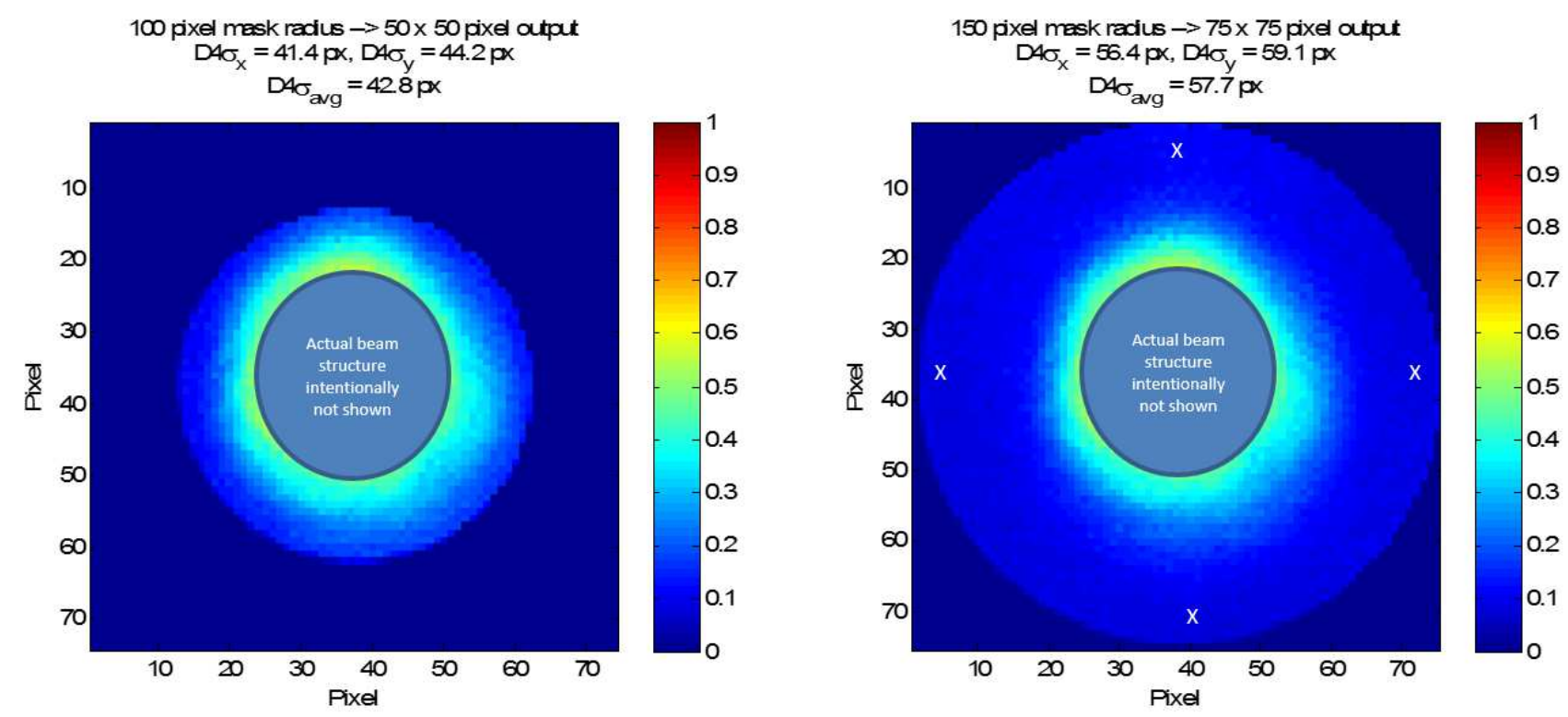

Figure 3. Intensity profiles that were reconstructed using different user-selected mask sizes on the same raw interferogram (the left image has been zero-padded to $75 \times 75$ pixels for easier comparison to the right image). For confidentially reasons the actual structure of the beam is not shown. The beam profile contains a distinct peak to which all intensities have been normalized. Note that a significant background intensity ( $\sim 10 \%$ of peak intensity) is retrieved within the mask region at locations where it was known that no actual beam energy was present (indicated by X's in right figure). As a result the deduced beam diameter is highly dependent on the (arbitrary) choice of mask size. Increasing the mask size by $50 \%$, led to an increase in beam size of $35 \%$. 

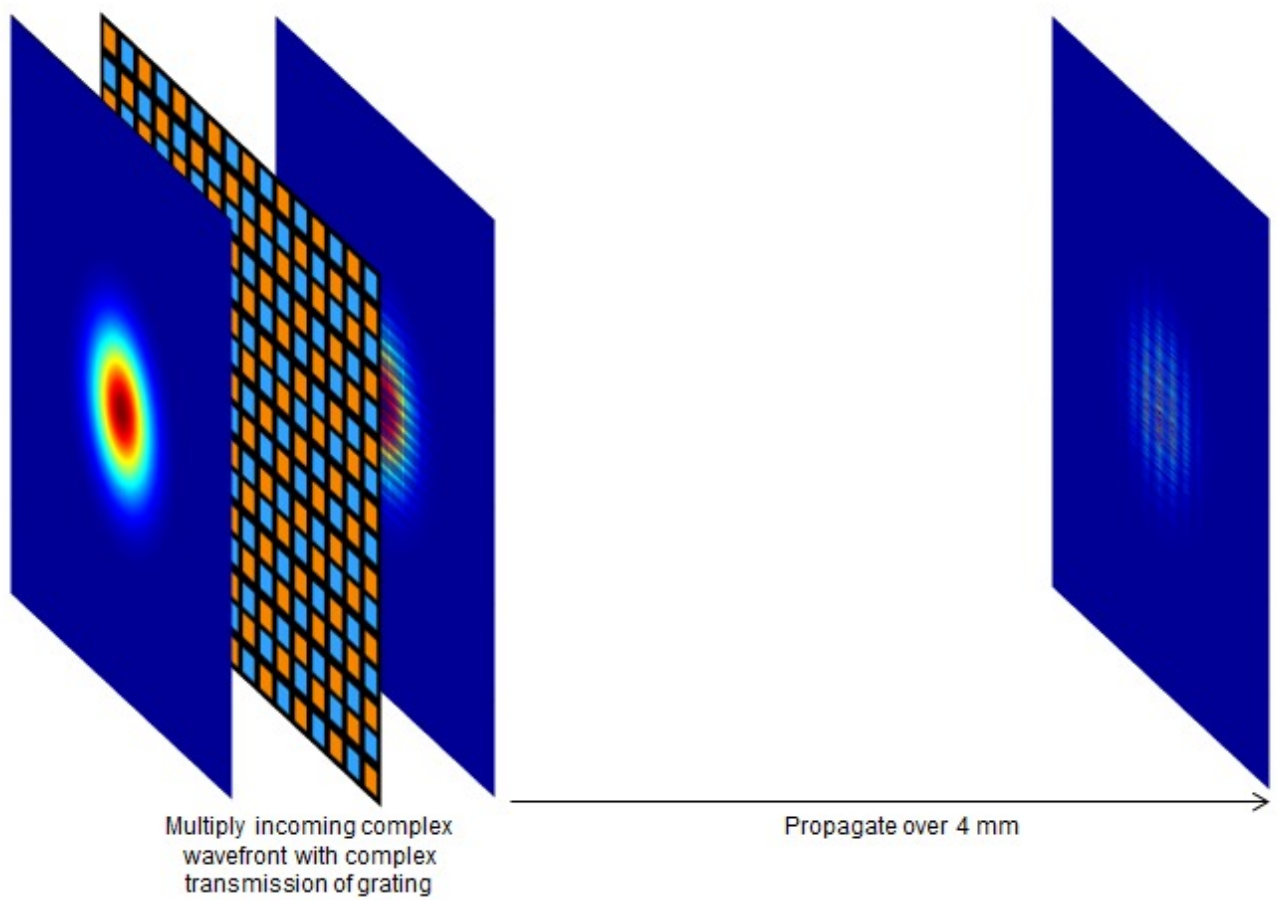

Propagate over $4 \mathrm{~mm}$

Figure 4. Schematic representation of the physical wavefront propagation that is used to determine the intensity impingent onto the QWLSI WFS imager.

\subsection{Physical wavefront propagation}

To determine the intensity distribution that is impingent onto the QWLSI WFS imager for a certain incoming wavefront, the complex wavefront is numerically propagated using Huygens-Fresnel, or first Rayleigh-Sommerfeld, type of propagation ${ }^{5}$.

This numerical propagation is schematically shown in Figure 4. As a first step the complex amplitude of the incoming beam is multiplied by the amplitude and phase transmittance of the QWLSI grating. In a second step free space propagation of the resultant wavefront is performed. If the complex amplitude directly after the grating is denoted by $p_{g}(x, y ; z=0)$, then, the resulting complex amplitude at the plane of the imager is given by: ${ }^{5}$

$$
p_{i}(x, y ; z)=p_{g}(x, y ; z=0) \otimes n(x, y ; z),
$$

where $\otimes$ denotes convolution and

$$
n(x, y ; z)=\frac{z \cdot \exp \left(i \frac{2 \pi}{\lambda} \sqrt{x^{2}+y^{2}+z^{2}}\right)}{x^{2}+y^{2}+z^{2}} .
$$

is the exact propagation kernel. Because a convolution is computationally expensive $p_{i}$ is calculated using

$$
p_{i}(x, y ; z)=\mathscr{F}^{-1}\left[\mathscr{F} \mathrm{p}_{\mathrm{g}}(\mathrm{x}, \mathrm{y} ; \mathrm{z}=0) \cdot \mathscr{F} \mathrm{n}(\mathrm{x}, \mathrm{y} ; \mathrm{z})\right],
$$

where the Fourier transforms ( $\mathscr{F}$ ) are performed using the Fast Fourier Transform (FFT) algorithm.

Figure 5 shows the calculated intensity that falls onto the imager when a $525 \mu \mathrm{m}$ diameter aperture is imaged onto the grating plane of the WFS. Although such a beam is much smaller than a beam that would normally be measured, using such a small beam clearly shows the higher-order diffraction due to the grating, and furthermore this case was very 


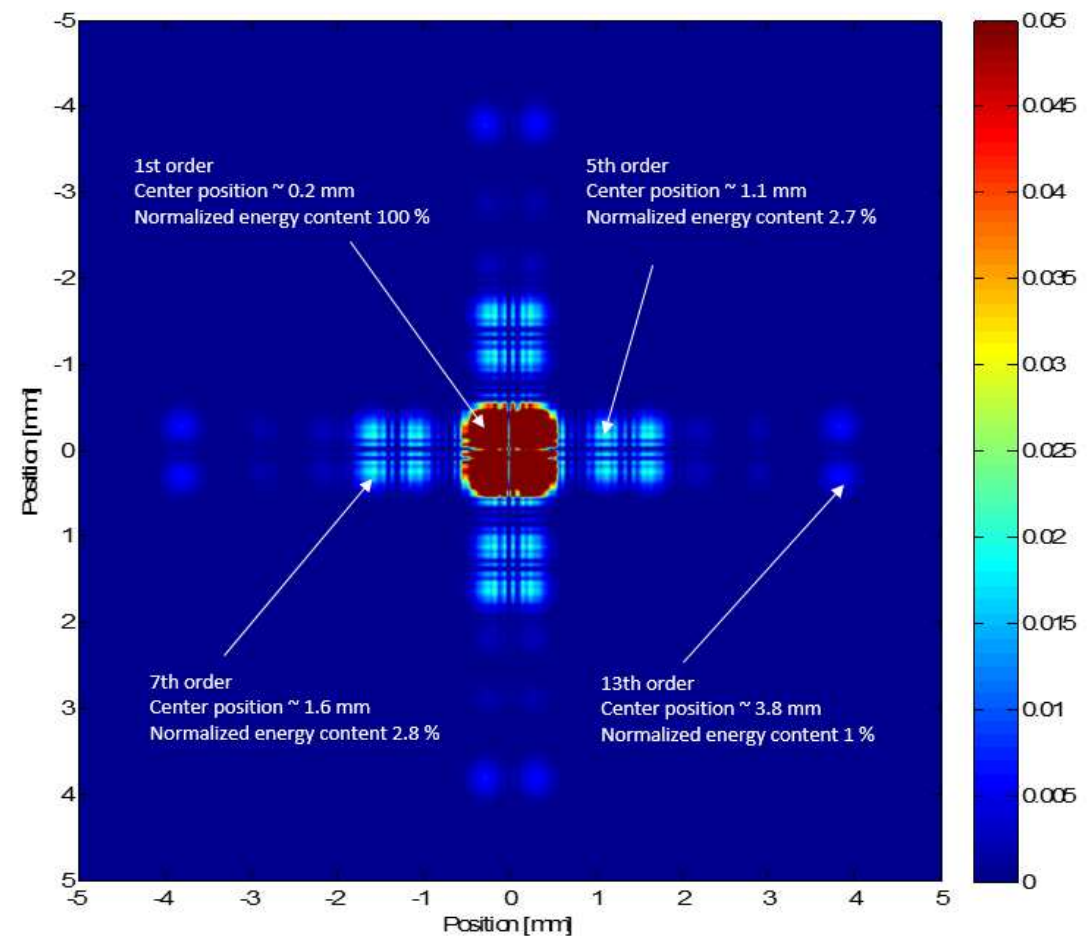

Figure 5. Simulated intensity that falls onto the QWLSI WFS imager when a $525 \mu \mathrm{m}$ diameter aperture is imaged onto the plane of the grating. Due to the limited size of the incoming beam, the energy due to multiple higher order diffraction is clearly visible. The dynamic range of the image has been adjusted such that the lower irradiance levels are observed (all intensities have been normalized to the peak intensity, and those higher than 5\% of the peak intensity are shown as 5\%). Also indicated are the location and normalized energy content of the $1^{\text {st }}, 5^{\text {th }}, 7^{\text {th }}$ and $13^{\text {th }}$ orders (as determined from this simulation).

interesting because experimental data of exactly the same situation was available for comparison. However, these measurements were performed by a third-party and we did not obtain timely approval to include these results in this paper. Nevertheless, aside from very low intensity level random noise, there is a 1-to-1 match between the measured and the simulated data. The magnitude and position of the higher orders, as well as the interference between neighboring orders, are in extreme close agreement. As such, our model has been shown to accurately describe the distortions induced by the QWLSI WFS.

From the simulated results (and related measurement data) shown in Figure 5, we found that the following diffraction orders are clearly visible in the far field: $1^{\text {st }}$ order $(100 \%), 5^{\text {th }}$ order $(2.7 \%), 7^{\text {th }}$ order $(2.8 \%)$ and $13^{\text {th }}$ order $(1 \%)$. The percentages between brackets indicate the energy content of each order, normalized to that contained in the first order. The angle $\alpha_{m}$ under which these orders propagate is given by the well-known grating equation:

$$
m \cdot \lambda=d^{\prime} \cdot \sin \alpha_{m},
$$

where $m$ denotes the order (i.e. 1, 5, 7, 13) and $d^{\prime}$ is the pitch of the phase grating (see Figure 2).

\subsection{Beam parameter propagation}

Although the physical wavefront propagation provides highly accurate results, it typically requires significant computing power and memory. There are cases where a less computationally expensive model is preferred.

When the D $4 \sigma$ beam diameter is of interest, an easy analytic propagation of the beam diameter throughout the QWLSI WFS is possible. For example, if we wish to consider the D4 $\sigma$ beam diameter along the $x$-coordinate axis, we can define 
$D 4 \sigma_{x}(z)$ as the $\mathrm{D} 4 \sigma$ beam diameter along the $x$-axis at an arbitrary longitudinal plane $z, D 4 \sigma_{x 0}$ as the minimum value of the $\mathrm{D} 4 \sigma$ beam diameter along the $x$-axis (i.e. the beam waist) and $z_{0 x}$ as the $z$-coordinate where this minimum occurs.

$D 4 \sigma_{x}$ can now be determined for any $z$ using: ${ }^{6}$

$$
D 4 \sigma_{x}(z)=D 4 \sigma_{x 0}\left[1+\left(\frac{4 \cdot(z-z 0 x) \cdot \lambda \cdot M_{x}^{2}}{\pi \cdot D 4 \sigma_{x 0}^{2}}\right)^{2}\right]^{1 / 2}
$$

where $M_{x}^{2}$ is the beam quality parameter in the $x$ direction, and in the remainder of this analysis $z_{0 x}$ is assumed to coincide with the plane of the grating.

Although the $M^{2}$ of the measured beam itself may be low (e.g. 1-1.5), the QWLSI WFS grating will induce significant amplitude and phase modulation, thereby effectively increasing the $M^{2}$ of the resulting wavefront.

The $M^{2}$ can be considered as the ratio between the actual $\mathrm{D} 4 \sigma$ diameter of the beam waist, and that which could have been minimally obtained, given the far-field divergence $\theta$ of the beam. Under the previously made assumption that the beam waist is imaged onto the grating plane, the actual size of the beam waist is thus the D4 $\sigma$ diameter of the beam $\left(D 4 \sigma_{\text {beam }}\right)$ impingent onto the QWLSI WFS. For the minimally obtainable (diffraction limited) D4 $\sigma$ beam diameter is commonly known that:

$$
D 4 \sigma_{\min }=\frac{2 \lambda}{\pi \theta} .
$$

From the above described definition of $M^{2}$ and Eq. (6) follows that:

$$
M^{2}=\frac{D 4 \sigma_{\text {beam }} \cdot \pi \cdot \theta}{2 \lambda} .
$$

In order to estimate the effective $M^{2}$ of the wavefront after the grating we thus need to determine the far field beam divergence after the grating.

Using our physical wavefront propagation model of the QWLSI WFS we determined for the far field divergence due to the grating $\theta \sim 0.32 \mathrm{rad}$.

Thus for example, when a beam with a D4 $\sigma$ beam diameter of $5 \mathrm{~mm}$ falls onto the QWLSI WFS grating, the D4 $\sigma$ beam diameter after the grating can be determined by Eq. 5 while using $M^{2}=237$.

\section{SENSOR-INDUCED BEAM SIZE ERROR}

\subsection{Incoming beam size dependence}

Using the physical wavefront propagation, the sensor-induced beam size error was examined for incoming Gaussian and top-hat beams of various diameters (at the nominal grating-to-imager distance of $4 \mathrm{~mm}$ ). Figure 6 shows the resulting intensity on the QWLSI WFS imager for a Gaussian and a top-hat beam $\left(D 4 \sigma_{\text {beam }}=3 \mathrm{~mm}\right)$.

For the same grating-to-imager distance the analytical propagation of the $\mathrm{D} 4 \sigma$ beam diameter was used to determine the beam size error, and its dependence on incoming beam size.

Figure 7 shows the outcome of these analyses. The physical wavefront propagation and analytic modelling are in close agreement, and clearly indicate that the sensor-induced beam size error can be very significant. For example, from Figures 6 and 7 can be seen that an incoming beam with $D 4 \sigma_{\text {beam }}=3 \mathrm{~mm}$ will result in a sensor-induced beam size error of $>30 \%$. 

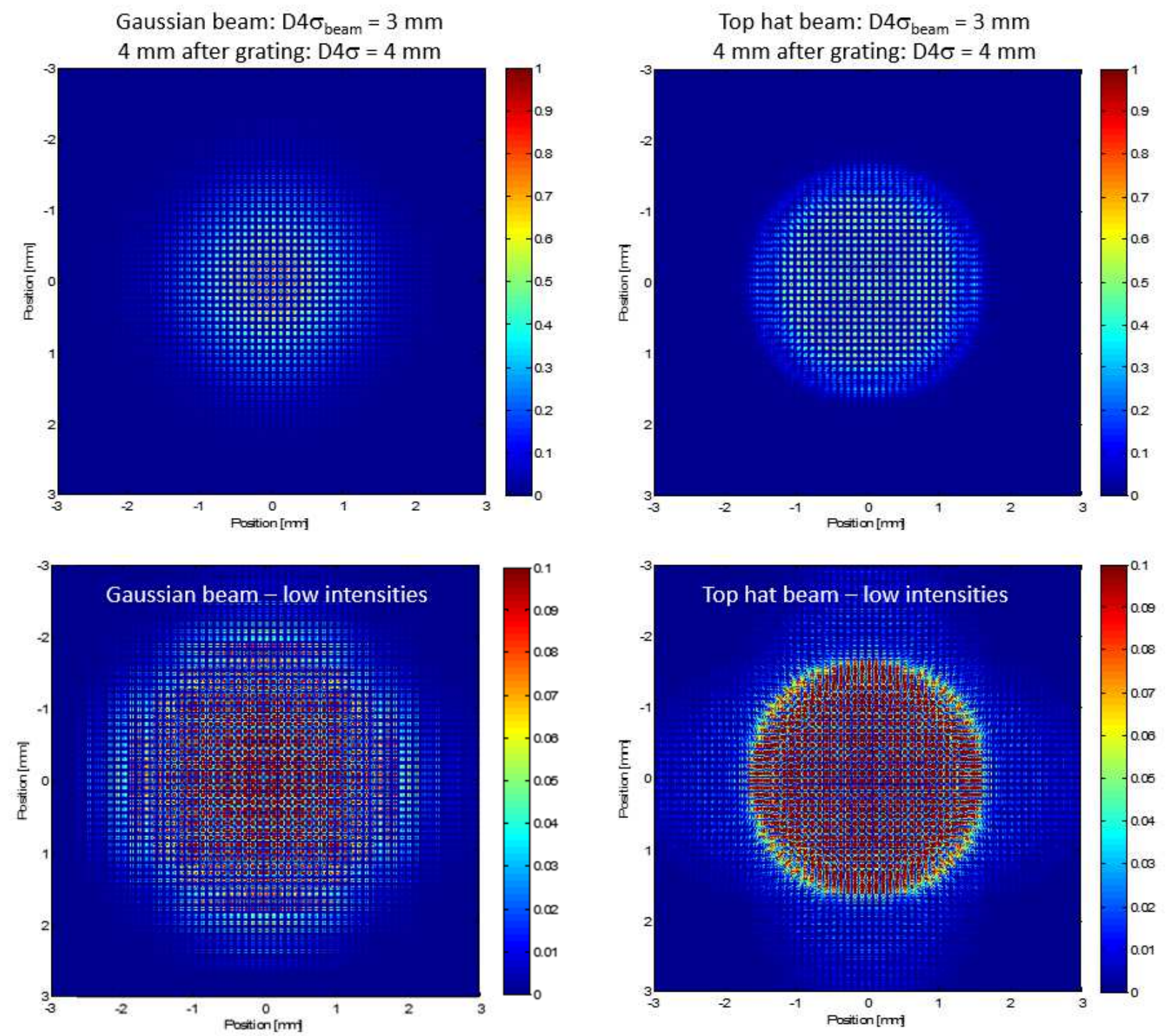

Figure 6. Simulated intensity that falls onto the QWLSI WFS imager given a Gaussian input beam (left) and top-hat beam (right). In the images on the bottom row the dynamic range of the image has been adjusted such that the lower irradiance levels are observed (all intensities have been normalized to the peak intensity, and those higher than $10 \%$ of the peak intensity are shown as $10 \%)$. 


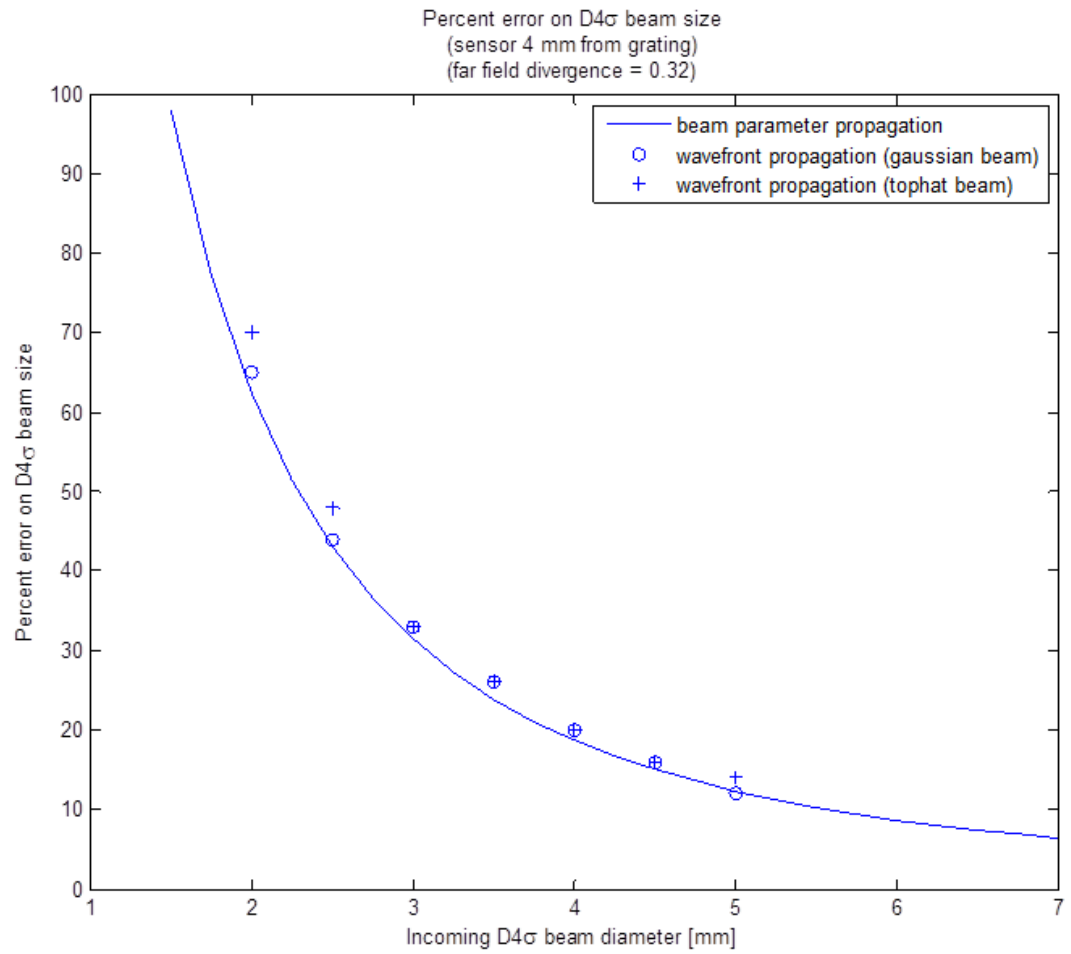

Figure 7. Beam size dependent sensor-induced beam size error. Depending on beam size, very significant errors can be introduced. Note that the beam parameter propagation shows very good agreement to the physical wavefront propagation.

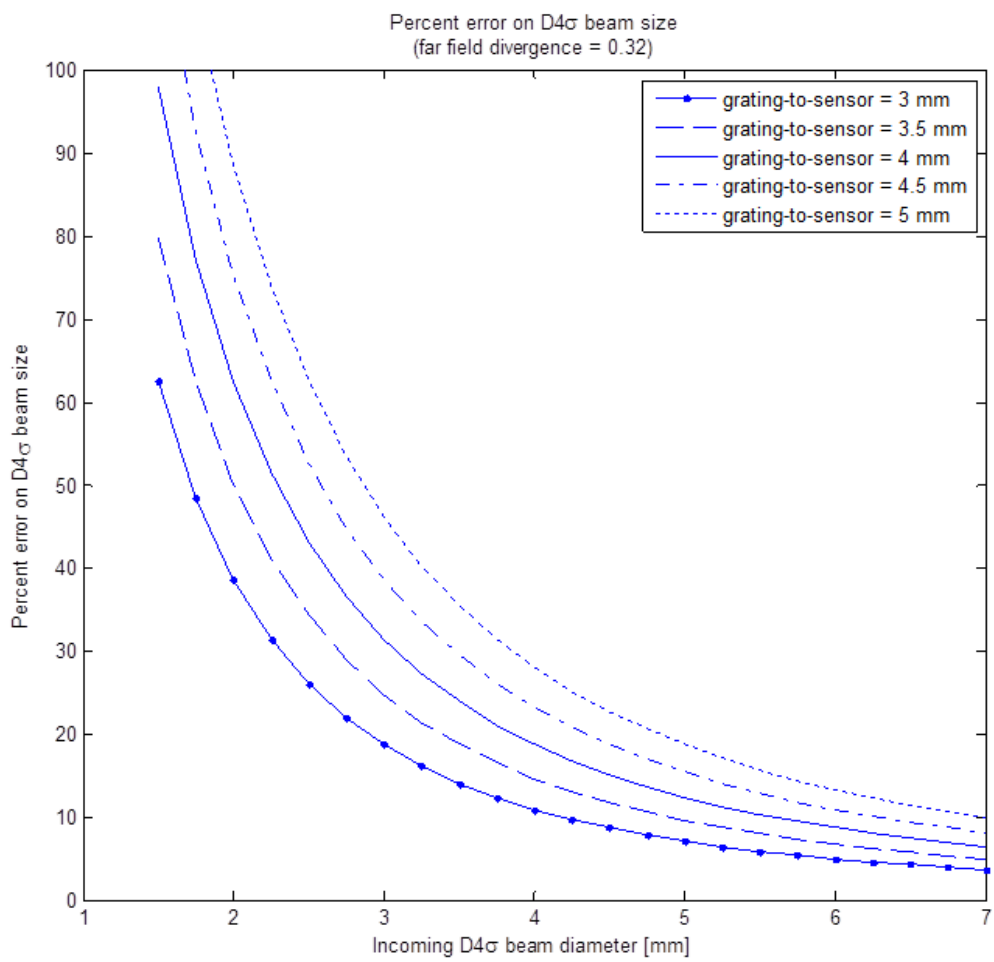

Figure 8. Sensitivity of sensor-induced beam size error to the distance between the grating and the QWLSI WFS imager. 


\subsection{Grating-to-sensor distance dependence}

In practice the grating-to-sensor distance has been seen to vary between $3.6-4 \mathrm{~mm}$. Figure 8 shows the sensitivity of the sensor-induced beam size error to the grating-to-sensor distance.

Particularly for small beam sizes it is important to use the actual grating-to-sensor distance when correcting for the sensor-induced beam size error. Using the nominally specified value may result in $\sim 10 \%$ residual error.

\subsection{Grating-geometry dependence}

As stated in Section 2.3., using our physical wavefront propagation model of the QWLSI WFS we determined for the far field divergence due to the grating $\theta \sim 0.32 \mathrm{rad}$.

In practice this far field divergence may vary. For instance, scatter from the transmission grating will likely lead to a further increase of the divergence. Also intentional changes on the grating geometry will impact the far field divergence.

Figure 9 shows the sensitivity of the sensor-induced beam size error to the far field divergence due to the grating. Since we do not have data on the expected variation of the far field divergence, it is hard to quantify realistic residual errors when assuming a nominal far field divergence.

\subsection{Wavelength dependence}

From Equation 4 can be readily seen that the angle under which a specific order is diffracted scales with $\lambda / d^{\prime}$. It is important to realize that as the ratio $\lambda / d$ ' increases, the increase in beam size due to (higher order) diffraction will become more significant.

From Section 2.1 we know that $d$ ' typically corresponds to two times the resolution that is obtained when the interferogram is processed using the software from the WFS manufacturer.

Table 1 provides an overview of the central wavelength and the associated reconstructed spatial resolution (assumed to be equal to $d$ ) for various commercially available QWLSI WFS ${ }^{2}$. The right hand column of Table 1 contains the sensor specific value for $\lambda / d^{\prime}$. There is a clear trend that as the central wavelength of a QWLSI WFS increases, the sensorinduced beam size error is expected to be larger.

Table 1. Wavelength dependence of angular extent of diffraction for various commercially available QWLSI WFS. ${ }^{2}$

\begin{tabular}{|c|c|c|c|}
\hline $\begin{array}{c}\text { Wavelength } \\
\text { range } \\
{[\mathrm{nm}]}\end{array}$ & $\begin{array}{c}\text { Central } \\
\text { wavelength } \\
\lambda \\
{[\mathrm{nm}]}\end{array}$ & $\begin{array}{c}\text { Spatial } \\
\text { resolution } d \\
{[\mu \mathrm{m}]}\end{array}$ & $\begin{array}{c}\text { Angular extent } \\
\text { of diffraction } \\
\lambda d d^{\prime} \\
{[\mathrm{nm} / \mu \mathrm{m}]}\end{array}$ \\
\hline $190-400$ & 295 & 32 & 4.6 \\
\hline $400-1100$ & 750 & 29.6 & 12.7 \\
\hline $1200-5000$ & 3100 & 60 & 25.8 \\
\hline $3000-5000$ & 4000 & 140 & 14.3 \\
\hline $8000-14000$ & 11000 & 140 & 39.3 \\
\hline $8000-14000$ & 11000 & 100 & 55 \\
\hline
\end{tabular}




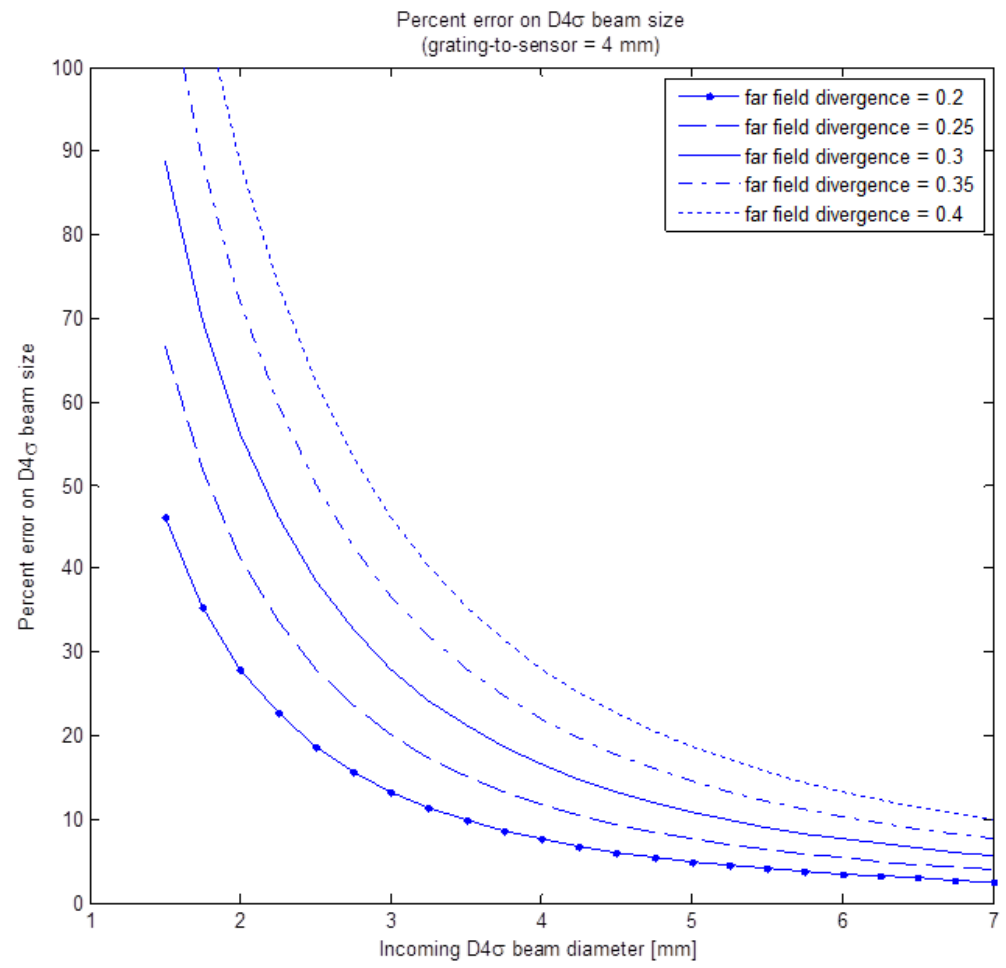

Figure 9. Sensitivity of sensor-induced beam size error to the far field divergence due to the grating.

\section{CONCLUSION AND RECOMMENDATIONS}

The raw interferogram from a QWLSI WFS is commonly used to determine (D4 $\sigma$ ) beam size, and other beam parameters. However, due to the physical principle of this type of sensor, the beam as measured by the QWLSI WFS imager can be significantly bigger than the incoming beam.

In this study we have provided an overview of the magnitude of this sensor-induced beam size error, and its sensitivity to various parameters such as incoming beam size, grating-to-sensor distance, grating geometry and wavelength. Given this knowledge it is possible to (largely) correct for the sensor-induced beam size error.

However, every correction method is prone to residual errors, and thus preventing errors from occurring is better than correcting for them afterwards. The best way to prevent the sensor-induced beam size error from occurring is to not rely on a QWLSI WFS for beam size measurements. However, in cases where only a QWLSI WFS is available, the following recommendations help to minimize the sensor-induced beam size error:

- Use as large a beam as the QWLSI WFS imager allows (while observing the requirements of ISO 11146)

- Try to avoid measuring multiple beams on a single QWLSI WFS

- A shorter grating-to-sensor distance will result in less beam spread, and is thus preferred from this perspective

- When different QWLSI WFS are available for the wavelength of interest, choose the one that will have the smallest angular extent of diffraction (i.e. smallest $\lambda d^{\prime}$ )

Additionally, since the position and relative energy content of the diffracted orders (e.g. see Figure 5) will effectively be convolved with the incoming beam profile to form the (low spatial frequency part of the) beam profile on the QWLSI WFS imager, it may be possible to retrieve the incoming beam profile from the QWLSI WFS interferogram. A separate 
study currently focusses on the development of (deconvolution-based) algorithms that allow for the robust retrieval of the incoming beam profile.

\section{REFERENCES}

[1] Velghe, S. et al, "On-axis and off-axis characterization of MWIR and LWIR Imaging Systems using Quadri Wave Interferometry,” Paper 8355-10 - SPIE Defense, Security and Sensing, Baltimore (2012)

[2] http://www.phasicscorp.com

[3] Primot, J. and Guérineau, N., "Extended Hartmann test based on the pseudoguiding property of a Hartmann mask completed by a phase chessboard," Appl. Opt. 39, 5715-5720 (2000).

[4] ISO 11146:2005, "Lasers and laser-related equipment - test methods for laser beam widths, divergence angles and beam propagation ratios"

[5] Goodman, J.W., "Introduction to Fourier Optics," Roberts \& Company Publishers (2005).

[6] Svelto, O., "Principles of Lasers," Plenum Press (1998). 